Refractive Index of Lithium Fluoride Ramp Compressed to $800 \mathrm{GPa}$

D. E. Fratanduono, T. R. Boehly, M. A. Barrios, D. D. Meyerhofer, J. H. Eggert, R. F. Smith, D. G. Hicks, P. M. Celliers, D. G. Braun, G. W. Collins

May 16, 2011

Journal of Applied Physics 
This document was prepared as an account of work sponsored by an agency of the United States government. Neither the United States government nor Lawrence Livermore National Security, LLC, nor any of their employees makes any warranty, expressed or implied, or assumes any legal liability or responsibility for the accuracy, completeness, or usefulness of any information, apparatus, product, or process disclosed, or represents that its use would not infringe privately owned rights. Reference herein to any specific commercial product, process, or service by trade name, trademark, manufacturer, or otherwise does not necessarily constitute or imply its endorsement, recommendation, or favoring by the United States government or Lawrence Livermore National Security, LLC. The views and opinions of authors expressed herein do not necessarily state or reflect those of the United States government or Lawrence Livermore National Security, LLC, and shall not be used for advertising or product endorsement purposes. 


\title{
Refractive Index of Lithium Fluoride Ramp Compressed to 800 GPa
}

D. E. Fratanduono*, T. R. Boehly, M. A. Barrios, and D. D. Meyerhofer* Laboratory for Laser Energetics, University of Rochester 250 East River Road, Rochester, NY 14623-1299, USA

*Also Department of Mechanical Engineering, University of Rochester 250 East River Road, Rochester, NY 14623-1299, USA

\author{
J. H. Eggert, R. F. Smith, D. G. Hicks, P. M. Celliers, D. G. Braun, and G. W. Collins \\ Lawrence Livermore National Laboratory, Livermore, CA 94550, USA
}

We report the highest pressure under which a transparent insulator has been observed. The refractive index of ramp-compressed lithium fluoride (LiF) is measured up to $800 \mathrm{GPa}$ pressure and is observed maintain its linear dependence on density. An effective single-oscillator model infers that the band gap closes monotonically with increasing density, indicating that metallization of LiF should occur at pressures above $4000 \mathrm{GPa}$, and that $\mathrm{LiF}$ should remain transparent at extremely high pressures.

\section{INTRODUCTION}

The transition of an insulator to a metal (metallization) at high compression is generally the result of pressure-induced closure of the band gap. ${ }^{1-4}$ Lithium fluoride's (LiF's) large band-gap and ionic crystalline structure produces its uniquely high ultraviolet transmissivity. Two rare-gas solids, $\mathrm{He}$ and $\mathrm{Ne}$, have anomalously high 
metallization pressures due to the predicted intershell band overlap, which is unique among monatomic materials. ${ }^{5} \mathrm{LiF}$ holds a rare position among binary compounds in that $\mathrm{Li}^{+}$and $\mathrm{F}^{-}$are iso-electronic with $\mathrm{He}$ and $\mathrm{Ne}$, respectively. Therefore, $\mathrm{LiF}$ is expected to have a uniquely high metallization pressure among large-band-gap solid insulators.

Under strong shock compression, the insulating-conducting transition is enhanced by the thermal promotion of electrons across the band gap. ${ }^{6}$ This is a result of high temperatures produced by high-pressure (>100 GPa) shock waves. A variety of materials has been shocked into conductive matter that is highly reflective at the shock front. ${ }^{6-10}$ LiF has been previously observed to be transparent when shocked to $\sim 115 \mathrm{GPa}$ (Refs. 8 and 11) and becomes reflective for shock pressures >500 GPa (Ref. 6).

Recently, ramp compression has been used to compress materials to pressures above $100 \mathrm{GPa}$, while keeping the temperature low compared to that of a shock wave. ${ }^{12}$ This work shows that $\mathrm{LiF}$ remains transparent when ramp compressed to $800 \mathrm{GPa}$; the highest pressure under which a transparent insulator has ever been observed. We use a new technique to measure its refractive index at pressures of 30 to $800 \mathrm{GPa}$. Early dynamic-compression experiments have shown the refractive index of various insulators to increase linearly with density at low pressure ( $\sim 100 \mathrm{GPa})$. The results presented here indicate that the linearity of the $\mathrm{LiF}$ refractive index increase over a larger density range than previously shown. This is expected since the electronic polarizabilites of large-bandgap insulators typically increase with compression. The results presented here are the highest-pressure refractive-index measurements seen to date.

Ramp-compressed $\mathrm{LiF}$ remains transparent at higher pressures than in shock compression because thermal excitation is insufficient to produce conduction electrons. 
As a result, at these high compressions we expect that only the effects of density are important and use this to infer the pressure-induced band-gap closure of $\mathrm{LiF}$ using a single-oscillator model. 13 Extrapolation of those results, although crude, indicates that ramp-compressed LiF may remain transparent to greater than $4000 \mathrm{GPa}$, well above the Goldhammer-Herzfeld criterion for metallization ( 2800 GPa) (Refs. 14-16). LiF will therefore continue to have technical utility as a diagnostic window for experiments at extreme pressures and the predicted band-gap closure provides important estimates for band-structure calculations.

In the present work, the refractive index of $\mathrm{LiF}$ was measured using ramp compression to $800 \mathrm{GPa}$. Diamond pistons were ramp compressed using the OMEGA laser. ${ }^{17}$ Diamond targets consisted of two sections; a free surface and a LiF window mounted on half of the rear surface. VISAR measurements were made at both sections to determine the refractive index. Section III discusses the relevant theory regarding the measurement technique followed by a discussion of the experimental method in Sec. IV. The experimental results are discussed in Sec. IV. Finally in Sec. V, an effectiveoscillator model is used to interpret the results providing an estimation of the band-gap closure.

\section{THEORY}

In many high-pressure experiments, the motion of a reflecting interface behind an optical window is detected by measuring Doppler shifts with a velocity interferometry system for any reflector (VISAR). 18 The reflected probe beam passes through the compression wave in the window and the observed Doppler shift, therefore, depends on 
the refractive index of the compressed window material. LiF is frequently used as an optical window because its transparency at high pressure allows in-situ measurements of samples confined by that window. 8,11 Hayes ${ }^{19}$ showed that for unsteady compression waves in windows, the true $\left(U_{\mathrm{t}}\right)$ and apparent $\left(U_{\mathrm{a}}\right)$ interface velocities (viewed through the compressed window) are directly related to the density-dependent refractive index of the window as

$$
\left.\frac{\mathrm{d} U_{\mathrm{a}}}{\mathrm{d} U_{\mathrm{t}}}\right|_{U_{\mathrm{t}}}=\left.\left(n-\rho \frac{\mathrm{d} n}{\mathrm{~d} \rho}\right)\right|_{U_{\mathrm{t}}} f(\rho),
$$

where the density $(\rho)$ and refractive index $(n)$ are evaluated as functions of $U_{\mathrm{t}}$. One can show that this gives $n(\rho)$ explicitly as

$$
n(\rho)=\rho\left[\frac{n_{0}}{\rho_{0}}-\int_{\rho_{0}}^{\rho} \frac{f\left(\rho^{\prime}\right)}{\rho^{\prime 2}} \mathrm{~d} \rho^{\prime}\right] .
$$

In this work $U_{\mathrm{a}}\left(U_{\mathrm{t}}\right)$ was measured using diamond as an impedance-match standard and the refractive index as a function of density was determined by solving Eq. (2).

\section{EXPERIMENTAL METHOD}

The targets consisted of a planar diamond piston with two sections: a free surface and one with an attached LiF window [see inset of Fig. 1(a)]. Planar compression waves, driven by direct laser ablation produced identical compression waves across the two 
sections (bare and $\mathrm{LiF}$ ) of the piston. The free-surface (bare diamond) velocity $\left(U_{\mathrm{fs}}\right)$ and the apparent interface (diamond-LiF) velocity $\left(U_{\mathrm{a}}\right)$ were measured simultaneously using VISAR. 18 The response of the free surface was used to infer the true velocity of the piston-window interface.

The method of characteristics 20 was used to determine the drive-pressure history applied to the target by propagating $U_{\mathrm{fs}}$ backward to the loading surface. The free-surface wave profile shown in Fig. 1 displays a distinct plateau at $2 \mu \mathrm{m} / \mathrm{ns}$ caused by the drop from the longitudinal to the bulk sound speed as the elastic limit of diamond is exceeded. In this experiment, as in previous experiments, ${ }^{12}$ the plateau shows a very constant velocity and the elastic limit is treated as a straightforward drop in the sound speed. Using that assumption, the calculated drive pressure is determined and then forward propagated to the diamond/LiF boundary, where pressures were impedance matched, thereby determining $U_{\mathrm{t}}$. The diamond-ramp wave response measured by Bradley et al., ${ }^{12}$ which includes diamond strength and the SESAME Table 7271 (Ref. 21) for LiF, was used. The LiF SESAME Table 7271 (Ref. 21) accounts for hydrostatic deformations while neglecting plastic deformations. The inclusion of a Steinberg-Guinan 22,23 strength model in the LiF equation of state (EOS) influenced the calculated interface pressure by $=1 \%$ prior to peak compression. As Ao et al. ${ }^{22}$ has shown, plastic effects in the interface velocity are of consequence only after peak compression. Analysis is terminated when interface yielding or "pullback" is observed, corresponding to $\sim 5.5 \mathrm{~ns}$ for the inset of Fig. 1. LiF strength effects were neglected since the error contribution was significantly less than other sources. 
The experiments were performed on the OMEGA Laser System. ${ }^{17}$ The ablation pressure was controlled by temporal shaping the drive laser pulse to produce compressions in $\mathrm{LiF}$ from 30 to $800 \mathrm{GPa}$. These drive pulses compressed the targets without creating shock waves in either the chemical-vapor-deposition (CVD) diamond pusher or LiF samples over the duration of the experiment.

Laser intensity scaled as $\sim t^{3}$ for pulse shape durations of 4 to $7 \mathrm{~ns}$. The diamond thickness for those experiments was $46 \mu \mathrm{m}( \pm 1 \mu \mathrm{m})$ and $100 \mu \mathrm{m}( \pm 3 \mu \mathrm{m})$, respectively. A 500- $\mu \mathrm{m}$-thick high-purity LiF window was mounted over the half of the rear of these diamond targets oriented with the $\langle 100\rangle$ axis along the pressure-loading direction. Samples were glued at the edges such that the compressed diamond released directly into the LiF where there was no glue. A $1000-\AA \AA$ titanium coating was applied to the diamond/LiF interface to increase the reflectivity for interferometer measurements. Three targets were constructed with gold layers ( 3 to $5 \mu \mathrm{m}$ thick) placed 10 to $15 \mu \mathrm{m}$ from the ablation surface to act as an $\mathrm{x}$-ray shield to ensure there was no effect from high-energy $\mathrm{x}$ rays passing through the target. No difference was observed in the results from targets with and without these shields.

\section{EXPERIMENTAL RESULT}

\section{A. Wave Profile Analysis}

A total of 17 shots were performed to determine the true and apparent velocities as described above. Each of the 17 continuous measurements is shown as an ensemble of like-colored points in Fig. 1(a). The measured apparent velocities were observed to monotonically increase with pressure with no observed discontinuities indicating a single 
phase of LiF existed to $800 \mathrm{GPa}$. This is consistent with recent ab-initio calculations performed by Smirnov. ${ }^{24}$ The pressures corresponding to these velocities were determined from the LiF SESAME $\operatorname{EOS}^{21}$ as shown on the top axis. In those experiments, a weak shock is initiated in the LiF window; this is treated as an initial characteristic whose parameters are determined from impedance matching with the Hugoniot. The effect of shock formation in the LiF window is examined in Sec. IIIB.

Seven additional targets employed $\sim 2 \mu \mathrm{m}$ of glue to fill the gap between the diamond and the LiF. At low pressures, the compressibility of the glue (and reverberations within it) caused the data to deviate from the targets with no glue between the $\mathrm{LiF}$ window and diamond. Once the glue "rang up" to higher pressure, the data followed the trend of targets with no glue-filled gap. Simulations indicate that after the glue rang up, corrections for the presence of $\sim 2-\mu \mathrm{m}$ gaps cause a shift of the true particlevelocity profiles after gap closure by $\sim 3 \mathrm{ps,} \mathrm{much} \mathrm{less} \mathrm{than} \mathrm{the} \mathrm{timing} \mathrm{accuracy} \mathrm{of} \mathrm{these}$ experiments.

The dominant errors in these calculations resulted from the precision of fringeshift measurements ( $2.5 \%$ of a fringe) and uncertainty in the diamond isentrope cited by Bradley et al. ${ }^{12}$ Measurements published by Hicks et al., 6 which were later re-examined by Celliers et al., ${ }^{25}$ are the only high-pressure measurements for LiF above $500 \mathrm{GPa}$ published to date. Comparison of measurements made by Hicks et al. ${ }^{26}$ at $\sim 1400 \mathrm{GPa}$ with the SESAME Table 7271 indicate an approximate $~ 7 \%$ error in pressure. Therefore, a $10 \%$ error in pressure is assumed for the $\mathrm{LiF}$ isentrope. ${ }^{22}$ The errors in the timing correlation between $U_{\mathrm{a}}$ and $U_{\mathrm{t}}$ was on the order of $47 \mathrm{ps}$ and $93 \mathrm{ps}$ for cameras 1 and 2, 
respectively, ${ }^{18}$ corresponding to uncertainties added in quadrature of the etalon delay ( $37 \mathrm{ps}$ and $78 \mathrm{ps}$ ) and nonuniformity in the drive planarity (5 pixels at sweep rate of $6 \mathrm{ps} / \mathrm{pixel}$ and $10 \mathrm{ps} / \mathrm{pixel})$. Systematic uncertainties are attributed to camera shear (6 and $10 \mathrm{ps}$ ), gap correction $\sim 1 \mathrm{ps}$ ( $33 \%$ of the calculated temporal shift), and the neglect of $\mathrm{LiF}$ material strength $(=1 \%$ change in peak interface pressure). Systematic uncertainties are estimated to be $5 \%$ of the total uncertainty and therefore neglected. Random errors were propagated through the method of characteristics using a Monte Carlo procedure that propagates uncertainties randomly chosen from a normal distribution. ${ }^{26}$

Steep gradients in the measured velocity profiles account for the large errors observed for single shots in Fig. 1(a). These were reduced by using a ramp compression that rose more gradually, albeit to lower pressures. Streaked optical pyrometer 27 measurements of $\mathrm{LiF}$ were dominated by thermal emission from the diamond anvil observed through the LiF. Comparison of the self-emission from the diamond-free surface and the diamond/LiF interface suggest that the $\mathrm{LiF}$ window temperature remained below $1000 \mathrm{~K}$ for all cases.

Figure 1(b) shows the weighted mean (black points) of the data from Fig. 1(a) using the associated errors discussed above. The large errors between 700 to $800 \mathrm{GPa}$ occur because only a single experiment reached those pressures. The dashed line is the linear portion of a fit performed to that weighted mean (see below). Shock-wave data taken from Wise and Chhabildas 8 and LaLone et al. ${ }^{11}$ are shown up to $115 \mathrm{GPa}$ as yellow and red squares, respectively.

A second-order orthogonal polynomial regression 25,26 was to fit the $U_{\mathrm{t}}[\mathrm{km} / \mathrm{s}]$ and $U_{\mathrm{a}}[\mathrm{km} / \mathrm{s}]$ particle velocities: $U_{\mathrm{a}}\left(U_{\mathrm{t}}\right)=a_{0}+a_{1}\left(U_{\mathrm{t}}-\beta\right)+a_{2}\left(U_{\mathrm{t}}-\gamma_{1}\right)\left(U_{\mathrm{t}}-\gamma_{2}\right)$, where 
$\beta=2.41 \mathrm{~km} / \mathrm{s}, \gamma_{1}=0.713 \mathrm{~km} / \mathrm{s}$, and $\gamma_{2}=9.53 \mathrm{~km} / \mathrm{s}$ are determined by the distribution of $U_{\mathrm{t}}$ in the data being fit. Since these parameters depend on the independent variable, errors are not assigned. Coefficients $\left(a_{0}, a_{1}\right.$, and $\left.a_{2}\right)$ are determined from a weighted $\chi^{2}$ minimization. In the orthogonal fit, the value of each coefficient is independent of higherorder terms, diagonalizing the covariance. $U_{\mathrm{t}}$ is considered the independent variable and $U_{\mathrm{a}}$ the dependent one with a standard deviation $\sigma_{U_{\mathrm{a}}}^{2}=\left(\delta U_{\mathrm{a}}\right)^{2}+\left(1.28 \delta U_{\mathrm{t}}\right)^{2} . \delta U_{\mathrm{a}}$ is the error associated with the measured interface velocity and $\delta U_{\mathrm{t}}$ is the error determined by Monte Carlo simulations, which is weighted by 1.28 to account for the estimated slope. Errors determined in the orthogonal fitting coefficients underestimate deviations observed within the data. This was observed when shots were removed at random and orthogonal fitting was performed. Coefficient errors were determined by standard deviations calculated from 100 discrete shot groupings of the 24 experiments: $a_{0}=$ $3.0634[\mathrm{~km} / \mathrm{s}], a_{1}=1.2751 \pm 0.0082$, and $a_{2}=0.0008 \pm 0.0015[\mathrm{~s} / \mathrm{km}]$. Errors are not assigned to $a_{0}$ since it represents the centroid of the data. The slope $\left(a_{1}\right)$ dominates the determination of the refractive index [Eq. (1)] and contributions from the curvature $\left(a_{2}\right)$ are assumed negligible because of their relative size and the bounding of zero.

Equation (2) was used to calculate $n(\rho)$ [Fig. (2)] using the weighted mean (black line). The refractive index and density under standard conditions (1.3935 and $2.6380 \mathrm{~g} / \mathrm{cc}$ ) were used as the boundary. The refractive index determined from the orthogonal fitting parameters is

$$
n=1.275( \pm 0.008)+0.045( \pm 0.003) \rho,
$$


where the second-order term $\left(a_{2}\right)$ has been neglected. The results obtained are in agreement with high-precision shock results up to $20 \mathrm{GPa}$ (Ref. 11)

$$
n=1.277( \pm 0.002)+0.0443( \pm 0.0008) \rho,
$$

and diamond-anvil experiments 28

$$
n=1.25( \pm 0.03)+0.05( \pm 0.01) \rho .
$$

The effective polarizability $(\alpha)$ of LiF is calculated using the Clausius-Mossoti relation,

$$
\frac{1}{\rho} \frac{\varepsilon-1}{\varepsilon+2}=\frac{4 \pi N_{a}}{3} \alpha,
$$

where $\varepsilon$ is the dielectric function and $N_{a}$ is Avogadro's number. For these experiments, the LiF absorption was negligible indicating that the imaginary part of the refractive index was small or $\varepsilon \approx n^{2}$. Using Eq. (6) and this approximation, the effective polarizability is calculated and plotted as a function of compression in Fig. (3). The effective polarizability is observed to decrease with increasing pressure.

\section{B. LASNEX Simulations}


LASNEX $^{29}$ simulations were performed to address concerns regarding shock formation in the $\mathrm{LiF}$ window and $\mathrm{LiF}$ material strength. The arrival of the diamond elastic limit (EL) limit in the LiF window generates a weak shock. Compression waves in the $\mathrm{LiF}$ window may reflect off the shock front and the arrival of those waves at the $\mathrm{LiF}$ would violate the conditions required in the derivation of Eq. (1) (Ref. 19). Since the method of characteristics does not account for the potential of shock formation, LASNEX simulations were performed to verify the characteristic calculations. LASNEX calculations used a diamond EOS with a Steinberg-Guinan strength model to recover the EL $^{23}$ and LiF SESAME Table 7271 (Ref. 21). The pressure drive was applied $10 \mu \mathrm{m}$ inside the front surface to account for the material that is ablated by the laser driver. This applied pressure is estimated to best match the measured diamond free-surface velocity.

LASNEX simulations were performed on shot 56113 and the results are shown in Figs. 4(a) and 4(b). Figure 4(a) contains a plot of the diamond free-surface velocity measured using VISAR (blue) and the estimated velocity determined from LASNEX (black) for an approximate applied laser intensity. Figure (4a) shows that the LASNEX free-surface velocity correlates well with the measured free-surface velocity. The applied pressure drive that determines the free-surface velocity is then used to simulate the true interface velocity. The noticeable discrepancies between the LASNEX simulation and measure free-surface velocity at $3.9 \mathrm{~ns}$ and $4.2 \mathrm{~ns}$ is due to the limitations of SteinbergGuinan strength model. That model predicts a higher EL than observed in this study generating the noticeable two-wave structure. As observed by McWilliams et al. the diamond EL varies and these variations are the cause of the observed discrepancy. 30 
Figure 4(b) compares interface velocities calculated using both methods. The LASNEX-predicted true interface velocity (black) and the true interface velocity calculated using the method of characteristics (red) infer nearly identical velocities prior to peak compression. The excellent agreement indicates that either (a) shock formation does not occur in the LiF window or (b) the effects of shock formation under these conditions do not significantly perturb the interface velocity prior to peak compression. This indicates that the requirements on Eq. (1) are met and Eq. (1) remains valid for these experiments. Furthermore, simulations performed with and without a Steinberg-Guinan strength model differ by less than $1 \%$ in pressure.

\section{DISCUSSION}

The temperatures achieved in this study are significantly lower than temperatures for identical pressures along the principle Hugoniot. SESAME Table 7271 (Ref. 21) predicts the principle isentrope temperature at $400 \mathrm{GPa}$ and $800 \mathrm{GPa}$ to be $\sim 700 \mathrm{k}$ and $\sim 800 \mathrm{k}$, respectively. The temperature along the principle Hugoniot for those pressures

are significantly higher $(12,500 \mathrm{k}$ and $31,500 \mathrm{k})$. Molecular dynamic simulations 31 predict that the Hugoniot approaches the melt line at $\sim 150 \mathrm{GPa}$ at $\sim 3500 \mathrm{k}$. Quantum molecular dynamics simulations predict as LiF melts, it transitions from a large gap insulator into a reflective material and during this transition develops a nonlinear refractive index. ${ }^{32}$ The low temperatures achieved in this study prevent LiF from approaching melt, inhibiting the development of a nonlinear refractive index.

An effective-oscillator model ${ }^{13}$ was used to interpret the observed linear dependence of refractive index on density. The effective-oscillator model describes 
optical properties as a weighted average over the visible spectrum. It is a simplistic dielectric model that predicts the magnitude and dispersion of ionic and covalent substances. The refractive index is defined by two "average" parameters - the dispersion energy $\left(E_{\mathrm{d}}\right)$ and single-oscillator energy $\left(E_{0}\right) . E_{0}$ is empirically related ${ }^{13}$ to the lowest direct optical transition $E_{\mathrm{t}}$ defined as the excitonic or band gap. The single-oscillator energy represents an average energy gap where the direct and indirect gaps may be smaller or exceed that energy. 33

Wemple and DiDomenico ${ }^{13}$ have shown that frequency-dependent refractive index for covalent and ionic materials can be fit to

$$
n^{2}-1=\frac{E_{\mathrm{d}} E_{0}}{E_{0}^{2}-\mathrm{h}^{2} \omega^{2}},
$$

where $E_{\mathrm{d}}$ is the dispersion energy, $E_{0}$ is the single-oscillator energy, and $\hbar \omega$ is the photon energy. In a survey of over 100 solid and liquid insulators at ambient conditions, this model fit the frequency-dependent refractive index well. ${ }^{13}$

This model was applied to the pressure-induced closure of the $\mathrm{H}_{2}$ band gap over a large density range ${ }^{34-36}$ and successfully predicted the emergence of excitonic absorption in the visible spectrum. ${ }^{4}$ Those studies show the $\mathrm{H}_{2}$ exciton shifts from $14.5 \mathrm{eV}$ to $2 \mathrm{eV}$ with a slightly sublinear dependence on density over nearly 15 -fold compression. The effective-oscillator model has been applied to $\mathrm{H}_{2} \mathrm{O}$ ice, demonstrating a linear reduction in the band gap over 2.3 -fold compression. ${ }^{33}$ In that study, the 
dispersion was measured from 569 to $741 \mathrm{~nm}$ at pressures ranging from 0 to $120 \mathrm{GPa}$. At discrete pressures, the effective-oscillator parameters $\left(E_{\mathrm{d}}\right.$ and $\left.E_{0}\right)$ were determined. Zha et al. 33 found that $E_{\mathrm{d}}$ was independent of density (remained constant) and that the effective gap closed monotonically with density. Wemple and DiDomenico ${ }^{13}$ have shown that the ratio of the band-gap energy to the lowest direct optical transition $\left(E_{0} / E_{\mathrm{t}}\right)$ is constant for constant dispersion energy $\left(E_{\mathrm{d}}\right)$.

The effective-oscillator model was applied to the data presented here, where the ambient values of $E_{\mathrm{d}}$ and $E_{0}$ were determined by fitting the refractive index to measured values in the range $332 \mathrm{~nm}<\lambda<732 \mathrm{~nm}$, i.e., the wavelength region near the probe laser. ${ }^{16}$ Fixing $E_{\mathrm{d}}$ to its ambient value, $E_{0}$ is calculated as a function of density using Eq. (3), as shown in Fig. 5. For the alkali halides of $\mathrm{NaCl}$ structure, the average ratio of single-oscillator energy to direct band gap is $E_{0} / E_{\mathrm{t}}=1.36$. This effective band gap begins at the intense exciton observed at ambient pressure 37 and then decreases with rising pressure. Extrapolation of these results (a crude approximation) indicates that the band gap may close above $>4000 \mathrm{GPa}$. This suggests that $\mathrm{LiF}$ will remain transparent well above the Goldhammer-Herzfeld $(\mathrm{G}-\mathrm{H})$ criterion ( 2800 GPa) (Refs. 14-16). This difference is consistent with the observed behavior of $\mathrm{He}$ and $\mathrm{Ne}$ which exhibited abnormally high metallization at pressures $10 \times$ greater than predicted by the $\mathrm{G}-\mathrm{H}$ criterion. 5,38

\section{CONCLUSION}


Direct laser ablation was used to ramp compress LiF from 30 to $800 \mathrm{GPa}$. LiF was observed to remain transparent over this pressure range; this is the highest-pressure insulator ever observed. The apparent and true particle velocities were measured over this range, and were used to calculate the refractive index of compressed LiF, which was found to depend linearly on density. These are the highest-pressure measurements of refractive index to date and are used to infer the pressure-induced band-gap closure of compressed LiF using an effective-oscillator model. These measurements provide the VISAR correction factor up to $800 \mathrm{GPa}$. Extrapolation of these results suggests that $\mathrm{LiF}$ remains transparent to above greater than $4000 \mathrm{GPa}$ as long as the temperature remains sufficiently low. If true, $\mathrm{LiF}$ will prove to be a valuable window for extremely high pressure, ramp-compression experiments.

\section{ACKNOWLEDGMENT}

This work was supported by the U.S. Department of Energy Office of Inertial Confinement Fusion under Cooperative Agreement No. DE-FC52-08NA28302, the University of Rochester, and the New York State Energy Research and Development Authority. Prepared by LLNL under Contract DE-AC52-07NA27344.

\section{REFERENCES}

1. R. Reichlin, M. Ross, S. Martin, and K. A. Goettel, Phys. Rev. Lett. 56, 2858 (1986).

2. K. A. Goettel, J. H. Eggert, I. F. Silvera, and W. C. Moss, Phys. Rev. Lett. 62, 665 (1989). 
3. R. Reichlin, K. E. Brister, A. K. McMahan, M. Ross, S. Martin, Y. K. Vohra, and A. L. Ruoff, Phys. Rev. Lett. 62, 669 (1989).

4. P. Loubeyre, F. Occelli, and R. LeToullec, Nature 416, 613 (2002).

5. J. C. Boettger, Phys. Rev. B 33, 6788 (1986).

6. D. G. Hicks, P. M. Celliers, G. W. Collins, J. H. Eggert, and S. J. Moon, Phys. Rev. Lett. 91, 035502 (2003).

7. S. B. Kormer, Sov. Phys.-Usp. 11, 229 (1968).

8. J. L. Wise and L. C. Chhabildas (NTIS Order No. DE85015505), Sandia National Laboratory, Albuquerque, NM, Report SAND-85-0310C, NTIS Order No. DE85015505 (1985).

9. D. K. Bradley, J. H. Eggert, D. G. Hicks, P. M. Celliers, S. J. Moon, R. C. Cauble, and G. W. Collins, Phys. Rev. Lett. 93, 195506 (2004).

10. J. Eggert, S. Brygoo, P. Loubeyre, R. S. McWilliams, P. M. Celliers, D. G. Hicks, T. R. Boehly, R. Jeanloz, and G. W. Collins, Phys. Rev. Lett. 100, 124503 (2008).

11. B. M. LaLone, O. V. Fat'yanov, J. R. Asay, and Y. M. Gupta, J. Appl. Phys. 103, 093505 (2008).

12. D. K. Bradley, J. H. Eggert, R. F. Smith, S. T. Prisbrey, D. G. Hicks, D. G. Braun, J. Biener, A. V. Hamza, R. E. Rudd, and G. W. Collins, Phys. Rev. Lett. 102, 075503 (2009).

13. S. H. Wemple and M. DiDomenico, Jr., Phys. Rev. B 3, 1338 (1971).

14. A. Goldhammer, in Monatshefte für Mathematik (Teubner, Leipzig, Berlin, 1913), Vol. 27.

15. K. F. Herzfeld, Phys. Rev. 29, 701 (1927). 
16. E. M. Bass, ed. Handbook of Optics, 2nd ed., Volume II: Devices, Measurements, and Properties (McGraw-Hill, New York, 1994).

17. T. R. Boehly, D. L. Brown, R. S. Craxton, R. L. Keck, J. P. Knauer, J. H. Kelly, T. J. Kessler, S. A. Kumpan, S. J. Loucks, S. A. Letzring, F. J. Marshall, R. L. McCrory, S. F. B. Morse, W. Seka, J. M. Soures, and C. P. Verdon, Opt. Commun. 133, 495 (1997).

18. L. M. Barker and R. E. Hollenbach, J. Appl. Phys. 43, 4669 (1972).

19. D. Hayes, J. Appl. Phys. 89, 6484 (2001).

20. J. R. Maw, AIP Conf. Proc. 706, 1217 (2004).

21. S. Crockett and S. Rudin, Los Alamos National Laboratory, Los Alamos, NM, LA-UR-06-8401 (2006).

22. T. Ao, M. D. Knudson, J. R. Asay, and J.-P. Davis, J. Appl. Phys. 106, 103507 (2009).

23. D. J. Steinberg, S. G. Cochran, and M. W. Guinan, J. Appl. Phys. 51, 1498 (1980).

24. A. Smirnov, Phys. Rev. B 83, 014109 (2011).

25. P. M. Celliers, G. W. Collins, D. G. Hicks, and J. H. Eggert, J. Appl. Phys. 98, 113529 (2005).

26. P. R. Bevington and D. K. Robinson, Data Reduction and Error Analysis for the Physical Sciences, 3rd ed. (McGraw-Hill, Boston, 2003).

27. J. E. Miller, T. R. Boehly, A. Melchior, D. D. Meyerhofer, P. M. Celliers, J. H. Eggert, D. G. Hicks, C. M. Sorce, J. A. Oertel, and P. M. Emmel, Rev. Sci. Instrum. 78, 034903 (2007). 
28. N. M. Balzaretti and J. A. H. Da Jornada, J. Phys. Chem. Solids 57, 179 (1996).

29. G. B. Zimmerman and W. L. Kruer, Comments Plasma Phys. Control. Fusion 2, $51(1975)$

30. R. S. McWilliams, J. H. Eggert, D. G. Hicks, D. K. Bradley, P. M. Celliers, D. K. Spaulding, T. R. Boehly, G. W. Collins, and R. Jeanloz, Phys. Rev. B 81, 014111 (2010).

31. R. Boehler, M. Ross, and D. B. Boercker, Phys. Rev. Lett. 78, 4589 (1997).

32. Clérouin, Y. Laudernet, V. Recoules, and S. Mazevet, Phys. Rev. B 72, 155122 (2005).

33. C.-S. Zha, R. J. Hemley, S. A. Gramsch, H. Mao, and W. A. Bassett, J. Chem. Phys. 126, 074506 (2007).

34. J. van Straaten and I. F. Silvera, Phys. Rev. B 37, 6478 (1988).

35. A. García, M. L. Cohen, J. H. Eggert, F. Moshary, W. J. Evans, K. A. Goettel, and I. F. Silvera, Phys. Rev. B 45, 9709 (1992).

36. R. J. Hemley, M. Hanfland, and H. K. Mao, Nature 350, 488 (1991).

37. D. J. Mickish, A. B. Kunz, and T. C. Collins, Phys. Rev. B 9, 4461 (1974).

38. S. A. Khairallah and B. Militzer, Phys. Rev. Lett. 101, 106407 (2008). 


\section{FIGURE CAPTIONS}

FIG. 1. (a) Apparent and true velocity measurements for all experiments. Vacuum-gap and glue-gap experiments are distinguished by their corresponding colors. Inset shows the target diagram. (b) Weighted average of all shots. Orthogonal regression and previous shock data are included. 8,11 Inset shows the VISAR measurements from a single experiment.

FIG. 2. Refractive index as a function of density determined using the weighted average and linear orthogonal fit. Previous data shock is included. 8,11

FIG. 3. The effective polarizability determined from the Lorentz-Lorenz relation plotted as a function of density.

FIG. 4. Comparison of hydrocode simulations and the method of characteristics for shot 56113. (a) LASNEX ablation pressure is determined by matching the LASNEX freesurface velocity (black) with VISAR measurement (blue). (b) The ablation pressure calculated using LASNEX is then used to determine the true interface velocity. Comparison of the LASNEX results (black) and the method of characteristics (red) is shown.

FIG. 5. Density dependence of the single-oscillator model $\left(E_{t}\right)$. Extrapolation suggests that $\mathrm{LiF}$ may remain transparent to pressure above $4000 \mathrm{GPa}$. 